\title{
Esthetic rehabilitation of anterior teeth with different thicknesses of porcelain laminate veneers: An 8-year follow-up clinical evaluation
}

\author{
Tassiana Cançado Melo Sá ${ }^{1}$, Monize Ferreira Figueiredo de Carvalho ${ }^{1}$, \\ Julio Celso M. de Sá ${ }^{2}$, Claudia Silami Magalhães ${ }^{1}$, Allyson Nogueira Moreira ${ }^{1}$, \\ Monica Yamauti ${ }^{1}$
}

Correspondence: Dr. Monica Yamauti

Email:myamauti@gmail.com

\begin{abstract}
'Department of Restorative Dentistry, Faculty of Dentistry, Federal University of Minas Gerais, Belo Horizonte, Minas Gerais, Brazil,

2Department of Restorative Dentistry, Faculty of Dentistry, Newton Paiva University Center, Belo Horizonte, Minas Gerais, Brazil
\end{abstract}

\section{ABSTRACT}

This clinical report describes an 8-year follow-up evaluation using different thicknesses of porcelain laminate veneers of diastema and malformed anterior teeth. Minimally invasive treatment with no preparation or minimal reduction options could be considered and chosen based on the characteristics of each case. Laminate veneers with or without reduction can offer an excellent result regarding esthetics and function. Despite its failure without reduction, this treatment is indicated because it can be applied regardless of the structure of the teeth. At first, no tooth preparation with rotatory instruments was performed, and porcelain laminates of minimum thickness were made for the four maxillary anterior teeth. After 6 years, the adhesive interfaces were stained, and the patient was unsatisfied with the esthetics of her smile. Therefore, at that time, the professional decided to prepare the anterior teeth for porcelain laminate veneers. Diastema closure and/or correction of malformed anterior teeth using porcelain laminate veneers is a viable option for the clinician because it restores esthetic harmony. The patient was very pleased with the new laminate veneers.

Key words: Dental esthetic, dental laminate, veneers

\section{INTRODUCTION}

Porcelain laminate veneers have gained popularity ${ }^{[1]}$ and are now applied in daily practice. Their esthetics, durability, and biocompatibility have made them a viable option for the treatment of anterior teeth. ${ }^{[2]}$ Advances in materials such as glass ceramic, adhesive systems, and resin cement have allowed restorative dentistry to refocus on minimally invasive procedures. ${ }^{[3]}$

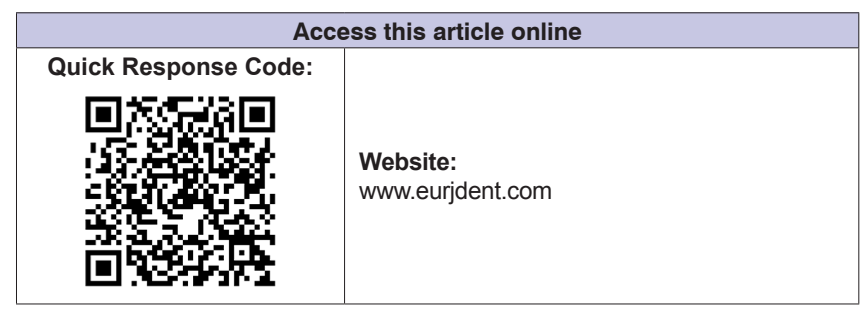

The presence of diastema or a malformed shape of the anterior teeth can be esthetically unsatisfactory, and the use of minimally invasive preparation-free porcelain veneers or a minimum reduction has become a viable treatment modality when conservative treatment is selected. ${ }^{[4]}$ The choice of minimal or no tooth preparation is a key factor in the prognosis. ${ }^{[5]}$

This is an open access journal, and articles are distributed under the terms of the Creative Commons Attribution-NonCommercial-ShareAlike 4.0 License, which allows others to remix, tweak, and build upon the work non-commercially, as long as appropriate credit is given and the new creations are licensed under the identical terms.

For reprints contact: reprints@medknow.com

How to cite this article: Melo Sá TC, Figueiredo de Carvalho MF, M. de Sá JC, Magalhães CS, MoreiraAN, Yamauti M. Esthetic rehabilitation of anterior teeth with different thicknesses of porcelain laminate veneers: An 8-year follow-up clinical evaluation. Eur J Dent 2018;12:590-3.

DOI: 10.4103/ejd.ejd_241_17 
Porcelain laminate veneers of minimum thickness provide satisfactory esthetic results and biocompatibility. ${ }^{[6]}$ Minimally invasive treatment options can be considered and chosen based on documentation, casts, and photographs. ${ }^{[7]}$ Indeed, some points should be considered to obtain success in the case: planning, type of preparation, selection of materials, and the continued maintenance of the restorations. ${ }^{[8]}$ This report describes an esthetic rehabilitation of anterior teeth with different thicknesses of porcelain laminate veneers and their longevity ${ }^{[8]}$

\section{CASE REPORT}

A 26-year-old female patient was unsatisfied with her smile. The intraoral examination showed diastemas between the maxillary incisors and a malformed lateral maxillary incisor [Figure 1a]. After the patient signed the document agreeing with the treatment plan, alginate impressions (Alginate; Jeltrate, Dentsply) were taken to obtain a diagnostic cast (Type IV dental stone, Herostone; Coltene). On the dental stone cast, diagnostic waxes were prepared to define the shape and form of teeth 11, 12, 21, and 22. From the waxes, a silicone guide was made. Diagnostic provisional restorations with direct composite resin (Filtek Z350, 3M ESPE) were prepared in situ to simulate the correct shape, function, and esthetic.

Porcelain laminates of minimum thickness were made for the four maxillary anterior teeth. No tooth preparation with rotatory instruments was performed. A displacement cord was used (Ultrapak Cord n\# 000; Ultradent products) to facilitate the impression procedure with additional silicone (Express XT, 3M, ESPE). The Vita shade guide (Vita Classical; Vita Zahnfabrik) was used to determine tooth color (A1).

Feldspathic porcelain laminate veneers were fabricated using a conventional laboratory refractory technique [Figure 1b]. The laminates were first etched with 10\% hydrofluoric acid (Porcelain Etchant; FGM Produtos Odontológicos) for $20 \mathrm{~s}$ and washed, dried, and silanized for $1 \mathrm{~min}$ (Monobond S; Ivoclar
Vivadent AG). The surface of the tooth was etched with $37 \%$ phosphoric acid (total etch; Ivoclar Vivadent AG) for $60 \mathrm{~s}$. One thin coat of hydrophobic resin (AdperScotchBond Multi-Purpose; 3M ESPE) was applied and polymerized for $10 \mathrm{~s}$. The laminates were luted with light-cured resin cement (Nexus 3; Kerr Corp). Light activation was performed with a light-emitting diode device (Radii-cal; SDI Ltda) for $40 \mathrm{~s}$ on each surface. The restorations were then examined for occlusal interferences [Figure 1c].

After 6 years, the adhesive interfaces were darkly stained, and the patient was unsatisfied with the esthetics [Figure 2a]. New impressions (Alginate; Jeltrate, Dentsply) were taken, dental stone casts were made, and provisional restorations were waxed-up to define the shape, form, and esthetic of the teeth.

A silicone replica of the diagnostic wax-up was prepared, and diagnostic restorations were placed with bis-acrylic interim material shade A1 (Protemp; 3M ESPE). Those restorations were used to evaluate the esthetics, function, and phonetics and to provide a guideline for tooth preparation. The teeth were then prepared. The labial surface reduction was designed with a dimension of $0.5 \mathrm{~mm}$ at the incisal third and middle third and $0.3 \mathrm{~mm}$ at the cervical third [Figure 2b]. An impression was taken as previously described.

Lithium disilicate glass ceramic (IPS e.max Press; Ivoclar Vivadent) porcelain laminate veneers were fabricated using the heat-press technique. The esthetic appearance of the veneers was evaluated using Variolink II Try-In (Ivoclar Vivadent AG). The ceramic veneers were luted in accordance with the Variolink Veneer [Figure 2c]. After 2 years, the veneers were intact without any chipping or discoloration [Figure 2d].

\section{DISCUSSION}

This clinical report demonstrates an approach to restoring maxillary anterior teeth that presented diastema and a malformation, using minimum dental preparation and adhesively bonded ceramic

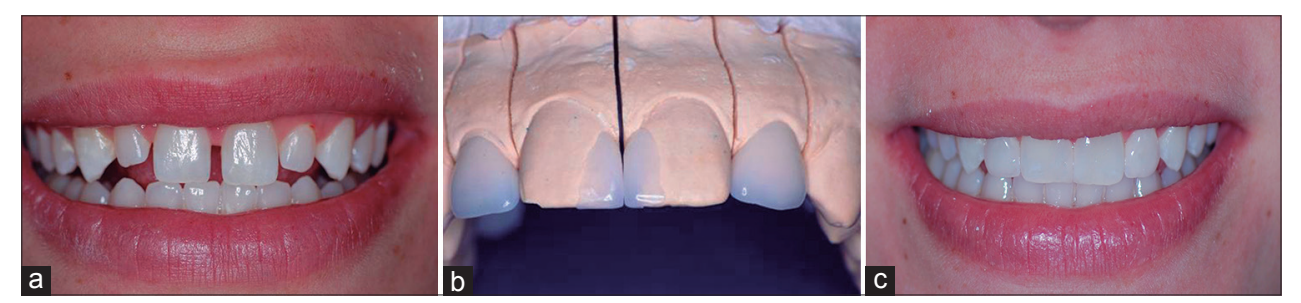

Figure 1: (a) Initial situation showing frontal view; (b) feldspathic ceramic laminate on the casts; (c) veneer after cementation 


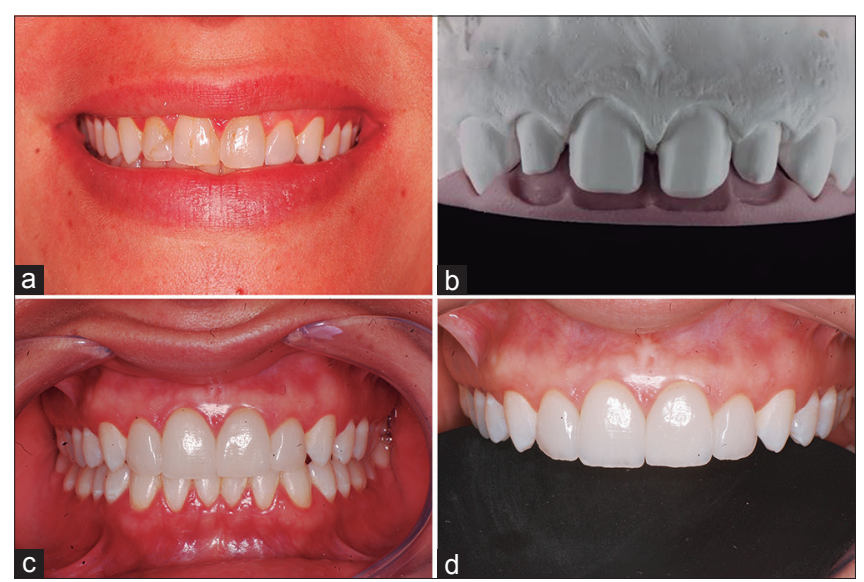

Figure 2: (a) Clinical aspect of the feldspathic ceramic laminate after 6 years; (b) minimal definitive preparation for ceramic veneers on anterior teeth; (c) facial view after cementation of ceramic veneers; (d) after 2 years

laminate veneers. Ceramic laminate veneers are more conservative than total crowns. The indications for ceramic laminate veneers include correction of tooth shape or position, changes in morphology of teeth with microdontia, and the presence of diastemas. ${ }^{[9]}$ Initially, the treatment for diastema closure did not involve tooth preparation for the use of very thin porcelain laminate veneers. The indication for noninvasive or minimally invasive preparation of laminate veneer includes teeth that require morphologic modifications and diastema closure. The severity and extent of any of these factors must be evaluated because they will determine the treatment goals, which have as much to do with restoring proper function as they do with esthetics. $^{[4]}$

However, after 6 years, the initial porcelain laminate veneers had become stained. Indeed, low survival of nonprepared laminate veneers is described compared with teeth that underwent a minimum reduction. ${ }^{[10]}$ Difficulty in creating a perfect transition between the tooth surface and the veneer is also reported for preparation-free veneers. ${ }^{[11]}$ The restoration bonds more tightly to prepared than to unprepared enamel. ${ }^{[1]}$ Furthermore, the median marginal gap and internal adaptation result in overlap between the veneer and the teeth. ${ }^{[10]}$

The right choice of resin cements and an appropriate cementation procedure are crucial in determining the life of resin-bonded ceramic restorations. ${ }^{[3]}$ The mechanical properties of resin cements are influenced by the type and composition of the resin matrix, and the stability of their color is linked to the absence of the aromatic tertiary amine component ${ }^{[8]}$ in light-cured resin cement. The first resin cement used in this case showed adequate polymerization and better results for microhardness. ${ }^{[12]}$

Nevertheless, after 6 years, this patient's adhesive interfaces were darkly stained, the patient was unsatisfied with the esthetics, and the teeth were then prepared. The failure of laminate veneers on unprepared teeth induced a new approach to this patient. The dental preparation restricted to the enamel was considered necessary for long-term success. ${ }^{[13]}$ Indeed, tooth reduction is important to produce a sufficient thickness of ceramic combined with a minimal thickness of luting composite, which can provide a favorable configuration of the restoration. ${ }^{[14]}$ Furthermore, preparation for laminate veneers with or without incisal coverage results in high survival rates and is consequently a safe option for the conservative treatment of anterior teeth. ${ }^{[15]}$

In the second stage of the treatment, a lithium disilicate glass ceramic was selected as the ceramic option. The feldspathic porcelain used in the first treatment was not chosen again because of the low mean strength values of this ceramic. ${ }^{[16]}$ However, lithium disilicate glass ceramics are commonly selected because of their optical properties, such as multiple translucencies and opacities, ${ }^{[3]}$ and adhesion to the tooth structure. ${ }^{[6]}$ Furthermore, this ceramic presents slower crack propagation, better resistance, and greater biaxial strength and fracture resistance. ${ }^{[17]}$ The use of conventional feldspathic ceramics, in view of their excellent optical characteristics, could afford optimum esthetic results when performed by an experienced technician. ${ }^{[8]}$

In addition, clinical failure of the adhesive interface should be considered. In observing the failure of different ceramic restorations, significantly fewer failures were detected when Variolink was used to fix the restorations, compared with other adhesive cements. ${ }^{[18]}$ However, the use of different luting cements cannot be considered as a predictor of the long-term survival of ceramic veneers. ${ }^{[19]}$ Regardless, for the luting agent used to fix ceramic veneers, the cement layer thickness should be as uniform and as thin as possible. ${ }^{[20]}$

For this patient, after removal of the restorations, complete crowns were deemed unnecessary. The patient also understood that she had to accept a compromised midline shift to avoid more invasive preparation of her teeth, including possible crowns and/or orthodontic treatment. 


\section{CONCLUSION}

The presence of diastema or malformed maxillary incisors can be esthetically unsatisfactory. The closure of diastema closure of the anterior teeth using porcelain laminate veneers is a viable option for the clinician because it restores esthetic harmony. Porcelain laminate veneers, with or without reduction, leads to an excellent result in terms of esthetics and function. Despite its failure without reduction, this treatment is indicated because it can be applied regardless of the structure of the teeth but will eventually need to be replaced.

\section{Declaration of patient consent}

The authors certify that they have obtained all appropriate patient consent forms. In the form, the patient has given her consent for her images and other clinical information to be reported in the journal. The patients understand that their names and initials will not be published and due efforts will be made to conceal their identity, but anonymity cannot be guaranteed.

\section{Financial support and sponsorship}

None.

\section{Conflicts of interest}

There are no conflicts of interest.

\section{REFERENCES}

1. Cötert HS, Dündar M, Oztürk B. The effect of various preparation designs on the survival of porcelain laminate veneers. J Adhes Dent 2009;11:405-11.

2. Fradeani M, Redemagni M, Corrado M. Porcelain laminate veneers: 6- to 12-year clinical evaluation - A retrospective study. Int J Periodontics Restorative Dent 2005;25:9-17.

3. Andrade OS, Ferreira LA, Borges GA, Adolfi D. Ultimate ceramic veneers: A laboratory-guided preparation technique for minimally invasive restorations. Am J Esthet Dent 2013;3:8-22.

4. Strassler HE. Minimally invasive porcelain veneers: Indications for a conservative esthetic dentistry treatment modality. Gen Dent 2007:55:686-94.

5. Stappert CF, Stathopoulou N, Gerds T, Strub JR. Survival rate and fracture strength of maxillary incisors, restored with different kinds of full veneers. J Oral Rehabil 2005;32:266-72.

6. da Cunha LF, Pedroche LO, Gonzaga CC, Furuse AY. Esthetic, occlusal, and periodontal rehabilitation of anterior teeth with minimum thickness porcelain laminate veneers. J Prosthet Dent 2014;112:1315-8.

7. Guzmán-Armstrong S, Maia RR. Conservative treatment planning in veneer replacement. J Prosthet Dent 2016;115:393-6.

8. Calamia JR, Calamia CS. Porcelain laminate veneers: Reasons for 25 years of success. Dent Clin North Am 2007;51:399-417, ix.

9. Fons-Font A, Solá-Ruíz MF, Granell-Ruíz M, Labaig-Rueda C, Martínez-González A. Choice of ceramic for use in treatments with porcelain laminate veneers. Med Oral Patol Oral Cir Bucal 2006;11:E297-302.

10. Schmitter M, Seydler B B. Minimally invasive lithium disilicate ceramic veneers fabricated using chairside CAD/CAM: A clinical report. J Prosthet Dent 2012;107:71-4.

11. Karagözoğlu İ, Toksavul S, Toman M 3D quantification of clinical marginal and internal gap of porcelain laminate veneers with minimal and without tooth preparation and 2-year clinical evaluation. Quintessence Int 2016;47:461-71.

12. Cho SH, Lopez A, Berzins DW, Prasad S, Ahn KW. Effect of different thicknesses of pressable ceramic veneers on polymerization of light-cured and dual-cured resin cements. J Contemp Dent Pract 2015;16:347-52.

13. Peumans M, Van Meerbeek B, Lambrechts P, Vanherle G. Porcelain veneers: A review of the literature. J Dent 2000;28:163-77.

14. Magne P, Kwon KR, Belser UC, Hodges JS, Douglas WH. Crack propensity of porcelain laminate veneers: A simulated operatory evaluation. J Prosthet Dent 1999;81:327-34.

15. Albanesi RB, Pigozzo MN, Sesma N, Laganá DC, Morimoto S. Incisal coverage or not in ceramic laminate veneers: A systematic review and meta-analysis. J Dent 2016;52:1-7.

16. Tinschert J, Zweza D, Marxa R, Anusavice KJ. Structural reliability of alumina-, feldspar-, leucite-, mica- and zirconia-based ceramics. J Dent 2000;28:529-35.

17. Gonzaga CC, Cesar PF, Miranda WG Jr., Yoshimura HN. Slow crack growth and reliability of dental ceramics. Dent Mater 2011;27:394-406.

18. Beier US, Kapferer I, Dumfahrt H. Clinical long-term evaluation and failure characteristics of 1,335 all-ceramic restorations. Int J Prosthodont 2012;25:70-8.

19. Radz GM. Minimum thickness anterior porcelain restorations. Dent Clin North Am 2011;55:353-70, ix.

20. Lin TM, Liu PR, Ramp LC, Essig ME, Givan DA, Pan YH, et al. Fracture resistance and marginal discrepancy of porcelain laminate veneers influenced by preparation design and restorative material in vitro. J Dent 2012;40:202-9. 